\title{
Fairness as a QoS Measure for Web Services
}

\author{
Stefano Bistarelli \\ Dipartimento di Scienze, Università "G. d'Annunzio" \\ Chieti-Pescara, Italy \\ Dipartimento di Matematica Informatica, \\ Università di Perugia, Italy \\ Istituto di Informatica e Telematica (CNR), \\ Pisa, Italy \\ bista@dipmat.unipg.it
}

\author{
Paola Campli \\ Dipartimento di Scienze, Università "G. d'Annunzio" \\ Chieti-Pescara, Italy \\ campli@sci.unich.it
}

\begin{abstract}
Service Oriented Architectures (SOAs) are component-based architectures, characterized by reusability, modularization and composition, usually offered by HTTP (web services) and often equipped with a Quality of Services (QoS) measure. In order to guarantee the fairness property to each client requesting a service, we propose a fair version of the (Soft) Concurrent Constraint language to deal with the negotiation phases of the Service Level Agreement (SLA) protocol.
\end{abstract}

\section{Introduction}

Service Oriented Architecture (SOAs) are the current technology for business to business interactions. SOAs are component-based architectures, characterized by reusability, modularization and composition, usually offered via HTTP (web services) and often equipped with a Quality of Service (QoS) measure. For example if the required service is routing a possible measure of quality could be bandwidth, cost or delay. However these metrics do not reflect the subjective criteria of individual users or groups. In this article, we consider fairness as a new parameter to be adopted in a QoS measure and we propose to use Soft Constraint to represent the QoS scores as suggested in [2].

When a user requests a service, he evaluates it with a QoS measure; we think that fairness is an important parameter to consider, as it directly affects the QoS value; therefore the service provider aims to guarantee a fair service in order to obtain a high QoS score.

We use (Soft) Concurrent Constraint (SCC) programming to model web services requests. Based on this formalism we introduce Fair Concurrent Constraint Programming, that uses an extension of the parallel operator which enables the provider to guarantee the fairness property to each client requesting a service. We consider soft constraints in order to define fairness as the level of preference associated to each constraint.

This paper is organized as follow: In section 2 we give a background of the Service Oriented Architecture; in section 3 we explain how to apply the soft concurrent constraint framework to the QoS negotiation; in section 4 we define the various notions of fairness; in section 5 we propose a fair version of the concurrent constraint (cc) language. Finally, in section 6 we model the SLA negotiation with the soft concurrent constraint language.

\section{Service Oriented Architecture}

A Service Oriented Architecture can be defined as a group of services, which communicate with each other [11]. The process of communication involves either simple data passing or it could involve two

M.H. ter Beek (Ed.): Young Researchers Workshop on Service-Oriented Computing 2009 (YR-SOC'09).

EPTCS 2, 2009, pp. 115-127 doi 10.4204/EPTCS.2.9 (c) S. Bistarelli \& P. Campli

This work is licensed under the Creative Commons Attribution License. 
or more services coordinating some activities. Some means of connecting services to each other are needed. Basic services, their descriptions, and basic operations (publication, discovery, selection, and binding) that produce or utilize such descriptions constitute the SOA foundation. The higher layers in the SOA pyramid provide additional support required for service composition and service management. The service composition layer encompasses necessary roles and functionality for the consolidation of multiple services into a single composite service. The resulting composite services may be used by service aggregators as components (basic services) in further service compositions or may be utilized as applications/solutions by service clients. The main characteristic of a SOAs are four:

- Coordination: Controls the execution of component services, and manages dataflow among them and to the output of the component service (by specifying workflow processes and using a workflow engine for runtime control of service execution).

- Monitoring: Subscribes to events or information produced by the component services, and publishes higher-level composite events (by filtering, summarizing, and correlating component events).

- Conformance: Ensures the integrity of the composite service by matching its parameter types with those of its components, imposes constraints on the component services (to ensure enforcement of business rules), and performing data fusion activities.

- Quality of Service (QoS) composition: Leverages, aggregates, and bundles the component's QoS to derive the composite QoS, including the composite service's overall cost, performance, security, authentication, privacy, (transactional) integrity, reliability, scalability, and availability.

Usually QoS metrics do not reflect the subjective criteria of individual users or groups. Our goal is to extend such QoS measure by adding Fairness as a new parameter in order to consider a subjective perception of the quality of the service. A subjective quality of the service represent the "ethical acceptance of the service" and it cannot be measured by the service provider just looking at one of the user, since all the users need to be considered together.

\section{Managing QoS Measures with Soft Concurrent Constraint}

The language we will use for web service interaction, composition and contracting is the Soft Concurrent Constraint [1]. Constraints has been used successfully to represent service interactions, contract requirements, and general services desiderata [10].

The aim of the paper is to apply Quality of Service (QoS) measures for Service Oriented Architectures considering Fairness as a new subjective attribute for QoS and for representing contracts and Service Level Agreements (SLAs) [9] in terms of constraint based languages. The notions of contract and SLAs are very important in SOC (service oriented computing) since they allow to describe the mutual interaction between communicating parties and to express properties related to the quality of service such as cost, performance, reliability and availability.

The use of a soft constraint framework permits to manage SOAs in a declarative fashion by considering together both the requirements/interfaces of each service and their QoS estimation [2]. C-semirings can represent several QoS attributes, while soft constraints represent the specification of each service to integrate: they link these measures to the resources spent in providing it, for instance, "the reliability is equal to $80 \%$ plus $5 \%$ for each other processor used to execute the service". This statement can be easily represented with a soft constraint where the number of processors corresponds to the $x$ variable, and the preference (i.e. reliability) level is given by the $5 x+80$ polynomial. In the same way we can consider and deal with the fairness property within QoS. 
A Soft Constraint-based formal language permit us to model and check QoS. In order to understand how to use the soft concurrent constraint framework we explain the key concepts related to it in the following sections.

\subsection{Concurrent Constraint Programming}

The concurrent constraint (cc) programming paradigm [14] concerns the behavior of a set of concurrent agents with a shared store, which is a conjunction of constraints ( relations among a specified set of variables). Each computation step possibly adds new constraints to the store. Thus information is monotonically added until all agents have evolved. The final store is a refinement of the initial one and it is the result of the computation. The concurrent agents communicate with the shared store, by either checking if it entails a given constraint (ask operation) or adding a new constraint to it (tell operation).

The syntax of a cc program is show in Table 1: $P$ is the class of programs, $F$ is the class of sequences of procedure declarations (or clauses), $A$ is the class of agents, $c$ ranges over constraints, and $x$ is a tuple of variables. The + combinator express nondeterminism. We also assume that, in $p(x):: A, \operatorname{vars}(A) \subseteq x$, where $\operatorname{vars}(A)$ is the set of all variables occurring free in agent $A$. In a program $P=F . A, A$ is the initial agent, to be executed in the context of the set of declarations $F$.

Table 1: cc syntax.

$$
\begin{aligned}
P & ::=F . A \\
F & ::=p(x):: A \mid F . F \\
A & ::=\operatorname{success} \mid \text { fail }|\operatorname{tell}(c) \rightarrow A| E|A \| A| \exists_{x} A \mid p(x) \\
E & ::=\operatorname{ask}(c) \rightarrow A \mid E+E
\end{aligned}
$$

The intuitive behavior of the agents is: agent "success" succeeds in one step; agent "fail" fails in one step; agent "ask $(c) \rightarrow A$ " checks whether constraint $c$ is entailed by the current store and then, if so, behaves like agent $A$. If $c$ is inconsistent with the current store, it fails, and otherwise it suspends, until $c$ is either entailed by the current store or is inconsistent with it; agent "ask $\left(c_{1}\right) \rightarrow A_{1}+\operatorname{ask}\left(c_{2}\right) \rightarrow A_{2}$ " may behave either like $A_{1}$ or like $A_{2}$ if both $c_{1}$ and $c_{2}$ are entailed by the current store, it behaves like $A_{i}$ if $c_{i}$ only is entailed, it suspends if both $c_{1}$ and $c_{2}$ are consistent with but not entailed by the current store, and it behaves like "ask $\left(c_{1}\right) \rightarrow A_{1}$ " whenever "ask $\left(c_{2}\right) \rightarrow A_{2}$ " fails (and vice versa); agent "tell $(c) \rightarrow A$ " adds constraint $c$ to the current store and then, if the resulting store is consistent, behaves like $A$, otherwise it fails; agent $A_{1} \| A_{2}$ behaves like $A_{1}$ and $A_{2}$ executing in parallel; agent $\exists_{x} A$ behaves like agent $A$, except that the variables in $x$ are local to $A ; p(x)$ is a call of procedure $p$.

Here is a brief description of the transition rules: 


$$
\begin{aligned}
& \langle\operatorname{tell}(c) \rightarrow A, \sigma\rangle \rightarrow\langle A, \sigma \otimes c\rangle \quad \text { tell } \\
& \frac{\sigma \vdash c,}{\langle A s k(c) \rightarrow A, \sigma\rangle \rightarrow\langle A, \sigma\rangle} \quad \text { ask } \\
& \frac{\left\langle A_{1}, \sigma\right\rangle \rightarrow\left\langle A_{1}^{\prime}, \sigma^{\prime}\right\rangle}{\left\langle A_{1} \| A_{2}, \sigma\right\rangle \rightarrow\left\langle A_{1}^{\prime} \| A_{2}, \sigma^{\prime}\right\rangle} \frac{\left\langle A_{1}, \sigma\right\rangle \rightarrow\left\langle A_{1}^{\prime}, \sigma^{\prime}\right\rangle}{\left\langle A_{2} \| A_{1}, \sigma\right\rangle \rightarrow\left\langle A_{2} \| A_{1}^{\prime}, \sigma^{\prime}\right\rangle} \quad \text { parallelism (1) } \\
& \frac{\left\langle A_{1}, \sigma\right\rangle \rightarrow\left\langle\text { success }, \sigma^{\prime}\right\rangle}{\left\langle A_{1} \| A_{2}, \sigma\right\rangle \rightarrow\left\langle A_{2}, \sigma^{\prime}\right\rangle} \frac{\left\langle A_{1}, \sigma\right\rangle \rightarrow\left\langle\text { success }, \sigma^{\prime}\right\rangle}{\left\langle A_{2} \| A_{1}, \sigma\right\rangle \rightarrow\left\langle A_{2}, \sigma^{\prime}\right\rangle} \quad \text { parallelism (2) } \\
& \frac{\left\langle E_{1}, \sigma\right\rangle \rightarrow\left\langle A_{1}, \sigma^{\prime}\right\rangle}{\left\langle E_{1}+E_{2}, \sigma\right\rangle \rightarrow\left\langle A_{1}, \sigma^{\prime}\right\rangle} \frac{\left\langle E_{1}, \sigma\right\rangle \rightarrow\left\langle A_{1}, \sigma^{\prime}\right\rangle}{\left\langle E_{2}+E_{1}, \sigma\right\rangle \rightarrow\left\langle A_{1}, \sigma^{\prime}\right\rangle} \quad \text { nondeterminism } \\
& \frac{\langle A[y / x], \sigma\rangle \rightarrow\left\langle A_{1}, \sigma^{\prime}\right\rangle}{\left\langle\exists_{x} A, \sigma\right\rangle \rightarrow\left\langle A_{1}, \sigma^{\prime}\right\rangle} \quad \text { hidden variables } \\
& \langle p(y), \sigma\rangle \rightarrow\langle A[y / x], \sigma\rangle \quad \text { where } \quad p(x):: A \quad \text { procedure call }
\end{aligned}
$$

\subsection{Soft Constraint}

Soft constraints generalize classical constraints by allowing several levels of satisfaction, and are able to express within the constraints many optimization criteria, and even the combination of some of them. The framework of soft constraints based on semirings [1] has shown to be general and expressive enough to deal with fuzziness, uncertainty, multiple criteria and also partial orders of preference levels.

The features of soft constraints could be useful in representing routing problems where an imprecise state information is given. Moreover, since QoS is only a specific application of a more general notion of Service Level Agreement (SLA), many applications could be enhanced by using such a framework. As an example consider E-commerce: here we are always looking for establishing an agreement between a merchant and a client. Also, all auction-based transactions need an agreement protocol.

Moreover, the possibility to embed soft constraints in a concurrent language environment is very useful to model agents interaction over the web. In fact, in this way not only we model the way constraints are solved, but we also have the possibility to describe the inter-agent protocols that are used to reach a common agreement.

For these reasons, in this paper we use the soft extension of the concurrent constraint language [14] with the possibility to handle soft constraints.

In the soft concurrent constraint, tell and ask agents are equipped with a preference (or consistency) threshold which is used to determine their success, failure, or suspension. The use of this thresholds yields an interesting and useful computational model, where it is possible to reason about the most convenient preference threshold to use to find the whole observable semantics of the language.

\subsection{Soft Constraint Problems}

A soft constraint may be seen as a constraint where each instantiations of its variables has an associated value from a partially ordered set. Combining constraints will then have to take into account such additional values, and thus the formalism has also to provide suitable operations for combination $(\times)$ 
and comparison (+) of tuples of values and constraints. This is why this formalization is based on the concept of c-semiring, which is just a set plus two operations.

c-semirings. A semiring is a tuple $\langle A,+, \times, 0,1\rangle$ such that:

- $A$ is a set and $0,1 \in A$;

- + is commutative, associative and 0 is its unit element;

- $\times$ is associative, distributes over,+ 1 is its unit element and 0 is its absorbing element.

A $c$-semiring is a semiring $\langle A,+, \times, 0,1\rangle$ such that: + is idempotent, 1 as its absorbing element and $\times$ is commutative.

Soft Constraint Systems. A soft constraint system is a tuple $C S=\langle S, D, V\rangle$ where $S$ is a c-semiring, $D$ is a finite set (the domain of the variables) and $V$ is an ordered set of variables. Given a semiring $S=$ $\langle A,+, \times, 0,1\rangle$ and a constraint system $C S=\langle S, D, V\rangle$, a constraint is a pair $\langle$ def, con $\rangle$ where con $\subseteq V$ and def: $D^{|c o n|} \rightarrow A$. Therefore, a constraint specifies a set of variables (the ones in con), and assigns to each tuple of values of these variables an element of the semiring. Consider two constraints $c_{1}=\left\langle\right.$ de $f_{1}$,con $\rangle$ and $c_{2}=\left\langle d e f_{2}, c o n\right\rangle$, with $\mid c o n \models k$. Then $c_{1} \sqsubseteq_{S} c_{2}$ if for all k-tuples $t, d e f_{1}(t) \leq_{S} d e f_{2}(t)$. The relation $\sqsubseteq_{S}$ is a partial order.

A soft constraint problem is a pair $\langle C$, con $\rangle$ where con $\subseteq V$ and $C$ is a set of constraints: con is the set of variables of interest for constraint set $C$, which however may concern also variables not in con. Note that a classical CSP is a SCSP where the chosen c-semiring is: $S_{C S P}=\langle\{$ false,true $\}, \vee, \wedge$, false, true $\rangle$. Fuzzy CSPs can instead be modeled in the SCSP framework by choosing the c-semiring $S_{F C S P}=$ $\langle[0,1], \max , \min , 0,1\rangle$.

Combining and projecting soft constraints. Given the two constraints $c_{1}=\left\langle\right.$ de $_{1}$, con $\left._{1}\right\rangle$ and $c_{2}=$ $\left\langle d e f_{2}\right.$, con $\left._{2}\right\rangle$, their combination $c_{1} \otimes c_{2}$ is the constraint $\langle$ def,con $\rangle$ defined by con $=\operatorname{con}_{1} \cup$ con $_{2}$ and $\operatorname{def}(t)=\operatorname{def} f_{1}\left(t \downarrow_{\text {con }_{1}}^{\text {con }}\right) \times d e f_{2}\left(t \downarrow_{\text {con }_{2}}^{\text {con }_{2}}\right)$, where $t \downarrow_{Y}^{X}$ denotes the tuple of values over the variables in $Y$, obtained by projecting tuple $t$ from $X$ to $Y$. In words, combining two constraints means building a new constraint involving all the variables of the original ones, and which associates to each tuple of domain values for such variables a semiring element which is obtained by multiplying the elements associated by the original constraints to the appropriate subtuples.

Given a constraint $c=\langle$ def,con $\rangle$ and a subset $I$ of $V$, the projection of $c$ over $I$, written $c \Downarrow_{I}$ is the constraint $\left\langle d e f^{\prime}, \operatorname{con}^{\prime}\right\rangle$ where $\operatorname{con}^{\prime}=\operatorname{con} \cap I$ and $d e f^{\prime}\left(t^{\prime}\right)=\sum_{t / t \downarrow_{I \cap \text { Inon }}=t^{\prime}} d e f(t)$. Informally, projecting means eliminating some variables. This is done by associating to each tuple over the remaining variables a semiring element which is the sum of the elements associated by the original constraint to all the extensions of this tuple over the eliminated variables. In short, combination is performed via the multiplicative operation of the semiring, and projection via the additive operation.

Solutions. The solution of an SCSP problem $\mathrm{P}=\langle C, \operatorname{con}\rangle$ is the constraint $\operatorname{Sol}(\mathrm{P})=(\otimes C) \Downarrow_{\text {con }}$. That is, we combine all constraints, and then project over the variables in con. In this way we get the constraint over con which is "induced" by the entire SCSP.

Sometimes it may be useful to find the semiring value of the optimal solutions. This is called the best level of consistency of an SCSP problem $P$ and it is defined by $\operatorname{blevel}(P)=\operatorname{Sol}(P) \Downarrow_{\emptyset}$. We also say that: $P$ is $\alpha$-consistent if $\operatorname{blevel}(P)=\alpha$; $P$ is consistent iff there exists $\alpha>_{S} 0$ such that $P$ is $\alpha$-consistent; $P$ is inconsistent if it is not consistent. 


\subsection{Soft Concurrent Constraint}

We briefly explain the main differences between CC and SCC [3]:

In the soft framework there are no notions of consistency and inconsistency. Instead, a notion of $\alpha$ consistency is introduced. This means that the syntax and semantics of the tell and ask agents have to be enriched with a threshold to specify when ask/tell agents have to fail, succeed or suspend.

Moreover, the notion of a thresholds $\alpha$ could be given pointwise w.r.t. each variable assignment (this is the notion of the following $\rightarrow_{\phi}$ ) or w.r.t. an overall consistency level of the constraint store (leading to the notion of $\rightarrow^{a}$ ).

Given a soft constraint system $\langle S, D, V\rangle$, the corresponding structure $C$, and any constraint $\phi \in C$, the syntax of $s c c$ agents is given in Table 2 .

Table 2: scc syntax.

$$
\begin{aligned}
& P::=F . A \\
& F \quad:=p(X):: A \mid F . F \\
& A \quad::=\operatorname{stop}\left|\operatorname{tell}(c) \rightarrow_{\phi} A\right| \operatorname{tell}(c) \rightarrow^{a} A|E| A \| A|\exists X . A| p(X) \\
& E \quad::=\operatorname{ask}(c) \rightarrow_{\phi} A\left|\operatorname{ask}(c) \rightarrow^{a} A\right| E+E
\end{aligned}
$$

The Operational Semantic for scc programs is given in the SOS style:

$$
\begin{aligned}
& \frac{(\sigma \otimes c) \Downarrow \emptyset \nless a}{\left\langle\operatorname{tell}(c) \rightarrow{ }^{a} A, \sigma\right\rangle \rightarrow\langle A, \sigma \otimes c\rangle} \\
& \frac{\sigma \otimes c \not \subset \phi}{\left\langle\operatorname{tell}(c) \rightarrow_{\phi} A, \sigma\right\rangle \rightarrow\langle A, \sigma \otimes c\rangle} \\
& \frac{\sigma \vdash c, \sigma \Downarrow \emptyset \nless a}{\left\langle a s k(c) \rightarrow^{a} A, \sigma\right\rangle \rightarrow\langle A, \sigma\rangle} \\
& \frac{\sigma \vdash c, \sigma \not \subset \phi}{\left\langle\operatorname{ask}(c) \rightarrow_{\phi} A, \sigma\right\rangle \rightarrow\langle A, \sigma\rangle} \\
& \frac{\left\langle A_{1}, \sigma\right\rangle \rightarrow\left\langle A_{1}^{\prime}, \sigma^{\prime}\right\rangle}{\left\langle A_{1} \| A_{2}, \sigma\right\rangle \rightarrow\left\langle A_{1}^{\prime} \| A_{2}, \sigma^{\prime}\right\rangle} \frac{\left\langle A_{1}, \sigma\right\rangle \rightarrow\left\langle A_{1}^{\prime}, \sigma^{\prime}\right\rangle}{\left\langle A_{2} \| A_{1}, \sigma\right\rangle \rightarrow\left\langle A_{2} \| A_{1}^{\prime}, \sigma^{\prime}\right\rangle} \\
& \frac{\left\langle A_{1}, \sigma\right\rangle \rightarrow\left\langle\text { success }, \sigma^{\prime}\right\rangle}{\left\langle A_{1} \| A_{2}, \sigma\right\rangle \rightarrow\left\langle A_{2}, \sigma^{\prime}\right\rangle} \frac{\left\langle A_{1}, \sigma\right\rangle \rightarrow\left\langle\text { success }, \sigma^{\prime}\right\rangle}{\left\langle A_{2} \| A_{1}, \sigma\right\rangle \rightarrow\left\langle A_{2}, \sigma^{\prime}\right\rangle} \\
& \frac{\left\langle E_{1}, \sigma\right\rangle \rightarrow\left\langle A_{1}, \sigma^{\prime}\right\rangle}{\left\langle E_{1}+E_{2}, \sigma\right\rangle \rightarrow\left\langle A_{1}, \sigma^{\prime}\right\rangle} \frac{\left\langle E_{1}, \sigma\right\rangle \rightarrow\left\langle A_{1}, \sigma^{\prime}\right\rangle}{\left\langle E_{2}+E_{1}, \sigma\right\rangle \rightarrow\left\langle A_{1}, \sigma^{\prime}\right\rangle} \quad \text { nondeterminism } \\
& \frac{\langle A[y / x], \sigma\rangle \rightarrow\left\langle A_{1}, \sigma^{\prime}\right\rangle}{\left\langle\exists_{x} A, \sigma\right\rangle \rightarrow\left\langle A_{1}, \sigma^{\prime}\right\rangle} \\
& \langle p(y), \sigma\rangle \rightarrow\langle A[y / x], \sigma\rangle \quad \text { when } \quad p(x):: A \quad \text { procedure call } \\
& \text { tell }
\end{aligned}
$$

Here is a brief description of the rules: 
Stop The stop agent succeeds in one step by transforming itself into terminal configuration success.

Valued-tell The valued-tell rule checks for the $\alpha$-consistency of the SCSP defined by the store $\sigma \cup c$. The rule can be applied only if the store $\sigma \cup c$ is $b$-consistent with $b \not \leq a$. In this case the agent evolves to the new agent $A$ over the store $\sigma \otimes c$. Note that different choices of the cut level $a$ will lead to different computations.

Tell The tell action is a finer check of the store. In this case, a pointwise comparison between the store $\sigma \otimes c$ and the constraint $\phi$ is performed. The idea is to perform an overall check of the store and to continue the computation only if there is the possibility to compute a solution not worse than $\phi$.

(Valued-)ask The semantics of the (valued-)ask is extended in a way similar to what we have done for the (valued-)tell action. This means that to apply the rule we need to check if the store $\sigma$ entails the constraint $c$ but also if the store is "consistent enough" w.r.t. the threshold ( $\phi$ or $a)$ set by the programmer.

Nondeterminism and parallelism The composition operators + and $\|$ are not modified w.r.t. the classical one: a parallel agent will succeed if all the agents succeed; a nondeterministic rule chooses any agent whose guard succeed. Note that at this stage no distinction between the don't know or don't care observational behaviour is made.

Hidden variables The semantics of the existential quantifier is similar to that of CCP by using the notion of freshness of the new variable added to the store.

Procedure calls The semantics of the procedure call is not modified w.r.t. the classical one. The only difference is the different use of the diagonal constraints to represent parameter passing.

The transition rules of scc could be applied to both eventual and atomic tell/ask interpretation. In fact, while the generic tell/ask rule represents an atomic behaviour, by setting $\phi=0$ or $a=0$ we obtain their eventual version.

Notice that, by using an eventual interpretation, the transition rules of the scc become the same as those of cc (with an eventual interpretation too). This happens since, in the eventual version, the tell/ask agent never checks for consistency and so the soft notion of $\alpha$-consistency does not play any role.

\section{Fairness as a Qos Measure for SOAs}

The fairness attribute we investigate represent the "ethical acceptance of the service" or the "perceived equity" of the service. This is clearly a "subjective" quality of the service and cannot be measured by the service provider just looking at one of the user, since all the user need to be considered together. Let us better explain our idea with an example. Suppose the service we consider is to have access to a table in a restaurant, and to be served by the waiter. A fair service will pay attention to the waiting time of the customer, and will try to provide the service with the "same" waiting time. Indeed, in order to score the quality of the service, a given user will not only evaluate only his (or her) own waiting time, but will also compare this waiting time with the waiting time of the other customers. This is what we mean for "perceived" quality of the service: if a user will wait some more, but will notice that no one will be served will be less disappointed than seeing other client sit at a table before him.

Fairness in computer science is often related to concurrent systems and to the guarantee that "eventually" each user will be served. Our goal is to obtain both perceived equity and fairness as defined in computer science. 
We can represent the users requiring a web service with the (soft) concurrent constraint framework where the concurrent agents can be seen as the clients requesting a service to the provider. Each client has a own measure of QoS that depends by the level of fairness that the user assigns to the service. The aim of the provider is instead to offer his service in a fair way, in order to maximize the QoS level of each customer.

To model this situation with our CC framework we need a fair method of selection of parallel agents: we propose the new parallel operator $\left(\|_{m}\right)$, in order to deal with a finite number of agents.

We clarify these notions with an example: suppose that more clients want to obtain a service (offered by a service provider) and this cannot be provided at the same time to all clients. The service provider has to select which agent can be satisfied first. In this case it needs a criterion for selecting one agent rather than another: this criterion should be as fair as possible to guarantee to all the customers the same level of perceived quality of the service. To model this scenario with the CC framework, we extend it with a new parallel operator, in order to obtain Fair Concurrent Constraint Programming.

\section{Fair Concurrent Constraint Programming}

In this section we present how to use a quantitative metric to provide a more accurate way to establish which of the agents involved in a parallel execution can succeed. The metric we use is the one proposed by a Fair carpooling scheduling algorithm [6], which is described more in detail below.

\subsection{The Fair Carpool Scheduling Algorithm}

Carpooling consists in sharing a car among a driver and one or more passengers to divide the costs of the trip. We want a scheduling algorithm that will be perceived as fair by all the members as to encourage their continued participation.

Let $U$ a value that represents the total cost of the trip. It is convenient to take $U$ to be the least common multiple of $[1,2, \ldots, m]$ where $m$ is the largest number of people who ever ride together at a time in the carpool. Since in a determined day the participans can be less than $m$, we define $n$ as the number of participants in the carpooling in a given day $(n \in[1 \ldots m])$.

Each day we calculate the passengers and driver's scores. This situation can be represented with a table which cols contains the members of the carpooling and inside the rows we insert the days; the intersection contains the score of each participant.

The first row has value zero for each member; in the following days the driver will increase his score of $U(n-1) / n$, while the remaining $n-1$ passengers decrease their score of $U / n$.

As proved in [6] this algorithm is fair; a finite computation is Carpooling-fair if for each enabled agent (or process) $A_{i}$ there is a number $N$ such that at each time $t$, the number of times that $A_{i}$ is executed $(E)$ differs from his ideal number of executions $I$ in absolute value than no more than $N$. The ideal number of executions represents the number of times each agent wishes to be executed. Formally we have: $\forall A_{i} \exists N$ s.t. $|E-I| \leq N$.

\subsection{The New Parallel Operator}

In order to obtain a Fair version of the parallel execution of a finite number of agents, we modify the parallel operator $\|$ in the CC semantic by replacing it with the $\|_{m}$ operator, which is able to deal with a set of $m$ agents. Since with the classical parallel operator only two concurrent agents are represented in 
the Parallelism rule, it is not possible to express fairness among more than 2 agents at the same level. The syntax of the $\mathrm{CC}$ language will be modified by replacing the $\|$ operator with $\|_{m}\left(A_{1}, \ldots, A_{m}\right)$. Moreover we develop the new transition rule, which is able to operate with the metrics of the carpooling algorithm. We introduce an array $\vec{k}$ and a set of conditions over $\vec{k},<\operatorname{cond}(k)>$ for the selection of the agent that we want to execute. The array $\vec{k}$ (with $m$ elements) allows us to keep track of the actions performed by each agent during the computational steps (we use the notation $\left\langle A_{i}, \vec{k}, \sigma\right\rangle$ instead of $\left\langle A_{i}, \sigma\right\rangle$ ). In this way we can associate a value $\vec{k}[i]$ to each agent $A_{i}$. The rule considers among the $m$ agents, the $n$ enabled agents (that we indicate with the notation: $A_{l_{1}} \rightarrow, \ldots, A_{l_{n}} \rightarrow$ ).

With reference to the carpooling algorithm, we represent the driver with the agent $A_{l_{i}}$, while the passengers are the remaining $(n-1)$ enabled agents.

Let $n \leq m$ represents the number of enabled agents and $A_{l_{i}}$ (with $i=[1, \ldots, n]$ ) the agent enabled and executed. $A_{l_{i}}$ increases his score of $U(n-1) / n$, while the $n-1$ enabled and not executed agents decrease their score of $U / n$. Initially all the $m$ elements of the array $\vec{k}$ are equal to 0 .

We define $\alpha_{n}=U(n-1) / n$ and $\beta_{n}=U / n$. These values are used in the following way: we add $\alpha_{n}$ to the previous value $\left(\vec{k}\left[l_{i}\right]\right)$ of the agent $A_{l_{i}}$; we subtract $\beta_{n}$ to the previous value $\left(\vec{k}\left[l_{j}\right]\right)$ of the other agents $A_{l j} \quad \forall j \in[1, \ldots, n], \quad j \neq i$.

In this case we use the condition $\left\langle\operatorname{cond}(k)>=\vec{k}\left[l_{i}\right] \leq \vec{k}\left[l_{j}\right]\right.$ that allows the (enabled) agent with a lower score to evolve. We update the scores in the variable $\vec{k}$ in the following way: the agent $A_{l_{i}}$ (that is the agent which performs the execution) increases his previous score of $\alpha_{n}$; the $(n-1)$ enabled agents decrease their score of $\beta_{n}$, while the scores of the not enabled agents remain unchanged. With the parameters provided by the carpooling algorithm we obtain the Carpooling-fairness rule:

Table 3: Carpooling fairness-parallelism (1) rule.

$$
\begin{gathered}
\vec{k}\left[l_{i}\right] \leq \vec{k}\left[l_{j}\right] \quad \forall \quad \begin{array}{l}
A_{l_{1}} \rightarrow, \ldots, A_{l_{n}} \rightarrow \\
j=1, \ldots, n \quad<A_{l i}, \sigma>\rightarrow<A_{l_{i}}^{\prime}, \sigma^{\prime}>
\end{array} \\
<\left\|_{m}\left(A_{1}, \ldots, A_{l_{i}}, \ldots, A_{m}\right), \vec{k}, \sigma>\rightarrow<\right\|_{m}\left(A_{1}, \ldots, A_{l_{i}}^{\prime}, \ldots, A_{m}\right), \vec{k}^{\prime}, \sigma^{\prime}> \\
\vec{k}[x]^{\prime}= \begin{cases}\vec{k}[x] & \text { if } x=1, \ldots, m \\
\vec{k}[x]+\alpha_{n} & \text { if } x=l_{i} \\
\vec{k}[x]-\beta_{n} & \text { if } x=l_{j} \quad j \neq i\end{cases}
\end{gathered}
$$

At each computation we select an agent according its value in the array $\vec{k}$ and then we update it in a way that at the following step, another agent has to be executed. This comply with the carpooling-fairness we defined.

We use the same criterion also for the Parallelism (2) rule:

If agent $A_{l_{i}}$ succeed, is deleted from the rule together with the corresponding element $\vec{k}\left[l_{i}\right]$, while the other values within the array $\vec{k}$ remain unchanged. Consequently the value $m$ of the operator $\|_{m}$ is continuously decreased of 1 every time an agent performs a success. We obtain therefore a final computation with a single agent and a single element on the array that reinstate the original rule: $\left\langle\|_{1}\left(A_{1}\right), \vec{k}[1], \sigma\right\rangle \rightarrow\left\langle A_{1}, \sigma\right\rangle$. 
Table 4: Carpooling fairness-parallelism (2) rule.

$$
\begin{aligned}
& A_{l_{1}} \rightarrow, \ldots, A_{l_{n}} \rightarrow \\
& \vec{k}\left[l_{i}\right] \leq \vec{k}\left[l_{j}\right] \quad \forall \quad j=1, \ldots, n \quad<A_{l i}, \sigma>\rightarrow<\text { success }, \sigma^{\prime}> \\
& <\left\|_{m}\left(A_{1}, \ldots, A_{l_{i}}, \ldots, A_{m}\right), \vec{k}, \sigma>\rightarrow<\right\|_{m-1}\left(A_{1}, \ldots, A_{l_{i}}-1, A_{l_{i}}+1, \ldots, A_{m}\right), \vec{k}^{\prime}, \sigma^{\prime}> \\
& \vec{k}[x]^{\prime}=\vec{k}[x] \quad \backslash \vec{k}\left[l_{i}\right]
\end{aligned}
$$

The new definition of the $\|_{m}$ operator permit us to maintain fairness step by step; in fact the provider of the service selects the agent according a well-defined fair criterion. Notice that as it is based on the classical CC framework, this actual extension of the parallel operator does not consider the preference level/fuzzyness of the constraint inserted into the store with a tell action.

In fact, to associate a level of preference to the constraints we need to use a semiring-based structure (see next section).

\section{Using Fair (S)CC for SLA Negotiation}

We use our fair extension of (S)CCP language to model a formal agreement. A Service Level Agreement [9] is an agreement regarding the guarantees of a web service. The parties involved in the SLA are the Service provider and the customers. The goal is to accomplish the requests of all the agents by satisfying their QoS requirements in a fair way, that is, we want that each customer is equally satisfied with respect to the other customers requesting the same service.

Suppose there are three customers (agents $A_{1}, A_{2}, A_{3}$ ) that want to request a service.

We model this scenario with the SCC language (therefore we use soft constraints) where each constraint has an associated preference level. We represent the requests of the service with the parallel execution of the three agents performing tell actions (as showed below) to the store:

$$
\begin{aligned}
& A_{1} \equiv \operatorname{tell}\left(c_{1}\right) \rightarrow^{a} \operatorname{tell}\left(c_{2}\right) \rightarrow^{a} \operatorname{tell}\left(c_{3}\right) \rightarrow^{a} \text { success } \\
& \| \\
& A_{2} \equiv \operatorname{tell}\left(c_{4}\right) \rightarrow^{a} \operatorname{tell}\left(c_{5}\right) \rightarrow^{a} \operatorname{success} \\
& \| \\
& A_{3} \equiv \operatorname{tell}\left(c_{6}\right) \rightarrow^{a} \operatorname{tell}\left(c_{7}\right) \rightarrow^{a} \operatorname{tell}\left(c_{8}\right) \rightarrow^{a} \operatorname{tell}\left(c_{9}\right) \rightarrow^{a} \text { success }
\end{aligned}
$$

In order to guarantee equity when is not possible for the service provider to offer the service contemporaneously, we have to select the agents according their level of preference; this is associated to each constraint and represents the subjective level of fairness requested by each agent.

We can use the new $\|_{m}$ operator to deal with soft constraints instead of crisp ones; we consider Soft Concurrent Constraint that permits to operate with preferences associated to the constraints. In this case, 
we want to select the agent with a lower level of preference; in the soft case the array $\vec{k}$ previously defined in the general rule contains soft constraints (unlike values used in the crisp case) added by the Agents which perform a tell action. This array is divided into sections: for each agent $A_{i}$ there is a section $\vec{k}[i]$ that contains the agent's constraints. These are combined through the $\otimes$ operator. For example, if Agent $\mathrm{i}$ performs the action: $\operatorname{tell}(c)$, we will modify the section $\vec{k}[i]$, obtaining $\vec{k}=\left[k_{1}, \ldots, k_{l_{i}} \otimes c, \ldots, k_{m}\right]$. Notice that in the soft case we don't use counters or values (as $\alpha$ and $\beta$ defined in the carpooling algorithm), but we just perform the combination of the constraints of the agent that succeed.

Below we show the modified rule of the parallelism operator that uses the array of soft constraints, and as condition Con $=\vec{k}\left[l_{i}\right] \sqsubseteq \vec{k}\left[l_{j}\right]$ :

$\|_{m}$ - parallelism (1) with soft constraints.

$$
\begin{gathered}
\vec{k}[i] \sqsubseteq \vec{k}[j] \quad \begin{array}{l}
A_{l_{1}} \rightarrow, \ldots, A_{l_{n}} \rightarrow \\
<A_{l i}, \sigma>\rightarrow<A_{l_{i}}^{\prime}, \sigma^{\prime}>
\end{array} \\
<\left\|_{m}\left(A_{1}, \ldots, A_{l_{i}}, \ldots, A_{m}\right), \vec{k}, \sigma>\rightarrow<\right\|_{m}\left(A_{1}, \ldots, A_{l_{i}}^{\prime}, \ldots, A_{m}\right), \vec{k}^{\prime}, \sigma^{\prime}> \\
\vec{k}[x]^{\prime} \begin{cases}\vec{k}[x] & \text { if } x=1, \ldots, m \\
\vec{k}[x] \otimes c & \text { if } x=l_{i} \\
\vec{k}[x] & \text { if } x=l_{j} \quad j \neq i\end{cases}
\end{gathered}
$$

This rule means that when the agent with the lower level of preference $\left(A_{l_{i}}\right)$ is selected (since the guard $\vec{k}\left[l_{i}\right] \sqsubseteq \vec{k}\left[l_{j}\right]$ permits it), it combines its constraint $(c)$ with the element $\vec{k}\left[l_{i}\right]$ obtaining $\vec{k}\left[l_{i}\right] \otimes c$; therefore its level of preference, that is his QoS measure, increases (this means that only the agent selected changes his preference level) and this constraint is inserted in the store $\sigma$; in this way we obtain a new store $\sigma^{\prime}$ that corresponds to the old store combined with the new constraint: $\sigma^{\prime}=\sigma \otimes c$.

Notice that if the computation $A_{l_{i}} \rightarrow A_{l_{i}}^{\prime}$ does not change the store, the computation evolves in a normal way, without affecting the vector $\vec{k}$. In the next step another agent is selected and its constraint combined with the previous constraints of the array $\vec{k}$, then it is added to the store and so on for the successive steps.

With the new transition rule, the provider will select the agent with the lower preference level, then it will combine its constraint through the $\otimes$ operator with the previous constraint in $\vec{k}$ and then to the store; in the example above, if at first step the enabled agent (with the lower soft constraint) is $A_{1}$, it will insert its constraint to the store, in the section $\sigma_{1}$, obtaining $\sigma_{1} \otimes c_{1}$; after this it can succeed to the following step (tell $\left.\left(c_{2}\right)\right)$. In the next step another agent is selected and its constraint added to the store and so on for the successive steps.

This rule is fair according the notions defined in section 4, because considers both the perceived equity, that is, each agent is served according a comparison with the preferences of the others, and fairness as the eventual execution of the agents; in fact, each enabled agent will be necessarily executed.

As each agent considers the service fair, this parameter will contribute to the increasing of the Quality of Service measure. 


\section{Related Work}

In this paper we modeled web services with the soft concurrent constraint language, considering fairness as a QoS parameter. In [5], where is studied air channel sharing in WLAN access networks, the authors propose fairness and quality of service as separeted issues in allocating wireless channels. In [4], is studied how a fair access in IEEE 802.11 bear its impact on quality of service. In [12] A Web services implementation is presented; the paper introduces a flexible framework to support fair non-repudiable B2B interactions based on a trusted delivery agent.

\section{Future Work}

In this article we used soft constraints as a fairness measure for QoS and we modeled the Service Level Agreement with the Soft Concurrent Constraint language. As future work we are going to apply the proposed formal technique to running web service example monitored for quality of service. In this way we can establish how the present approach on including fairness as a criteria actually influences the selection of a service by a service requester.

In economics the concept of fairness (or equity) is related instead to a function that represent the utility of each user. The concept of equity is in this case represented by some indexes that give a quantitative measure of fairness. There are various indexes for measuring economic inequality. The Gini coefficient is a measure of statistical dispersion most prominently used as a measure of inequality of income distribution or inequality of wealth distribution. It is defined as a ratio with values between 0 and 1: a low Gini coefficient indicates more equal income or wealth distribution, while a high Gini coefficient indicates more unequal distribution. We plan to focus on fairness by paying attention to these economic aspects and to the use of social welfare functions and indexes.

\section{References}

[1] S. Bistarelli (2004): Semirings for Soft Constraint Solving and Programming. Springer, Lecture Notes in Computer Science 2962, London, UK.

[2] S. Bistarelli \& F. Santini (2009): Soft Constraints for Quality Aspects in Service Oriented Architectures. In: M.H. ter Beek, editor: Proceedings of the 4th European Young Researchers Workshop on Service-Oriented Computing (YR-SOC'09). Electronic Proceedings in Theoretical Computer Science 2, pp. 51-65.

[3] S. Bistarelli, U. Montanari \& F. Rossi (2002): Soft Concurrent Constraint Programming. In: Proceedings of the 11th European Symposium on Programming Languages and Systems (ESOP'02). Lecture Notes in Computer Science 2305, Springer-Verlag, Berlin, pp. 53-67.

[4] M. Bredel \& M. Fidler (2008): Understanding Fairness and its Impact on Quality of Service in IEEE 802.11. CoRR abs/0808.3937.

[5] K. Dong-Young, E.-C. Park, C.-H. Choi \& J. So (2007): Improving Quality of Service and Assuring Fairness in WLAN Access Networks. IEEE Transactions on Mobile Computing 6(4), pp. 337-350.

[6] R. Fagin \& J.H. Williams (1983): A Fair Carpool Scheduling Algorithm. International Business Machine Corporation, pp. 133-139.

[7] N. Francez (1986): Fairness. Springer-Verlag, Berlin.

[8] IFIP (1998): IFIP WG 10.4 on dependable computing and fault tolerance. Technical Report, International Federation For Information Processing. 
[9] A. Keller \& H. Ludwig (2003): The WSLA framework: Specifying and monitoring service level agreements for web services. Journal of Network and Systems Management 11(1), pp. 57-81.

[10] A. Lazovik, M. Aiello \& R. Gennari (2005): Encoding Requests to Web Service Compositions as Constraints. In: Proceedings Constraint Programming (CP'05). Lecture Notes in Computer Science 3709, SpringerVerlag, Berlin, pp. 782-786.

[11] M.P. Papazoglou \& D. Georgakopoulos (2003): Service-oriented computing. Communications of the ACM 46(10).

[12] P. Robinson, N. Cook, \& S. Shrivastava (2005): Implementing Fair Non-repudiable Interactions with Web Services. In: Proceedings of the 9th International Enterprise Computing Conference (EDOC'05), IEEE Computer Society.

[13] V.A. Saraswat (1993): Concurrent Constraint Programming. The MIT Press, Cambridge, MA, USA.

[14] V.A. Saraswat \& M. Rinard (1990): Concurrent constraint programming. In: Proceedings of the 17th SIGPLAN-SIGACT symposium on Principles of Programming Languages (POPL'90), ACM Press, New York, pp. 232-245. 